\title{
Two-Channel Portable Biopotential Recording System Can Detect REM Sleep Behavioral Disorder: Validation Study with a Comparison of Polysomnography
}

\author{
Hiroshi Kataoka $\mathbb{D}$, Tsunenori Takatani, and Kazuma Sugie \\ Department of Neurology, Division of Central Clinical Laboratory, Nara Medical University, Kashihara, Nara, Japan \\ Correspondence should be addressed to Hiroshi Kataoka; hk55@naramed-u.ac.jp
}

Received 8 November 2021; Revised 4 February 2022; Accepted 5 February 2022; Published 24 February 2022

Academic Editor: Giovanni Mirabella

Copyright (c) 2022 Hiroshi Kataoka et al. This is an open access article distributed under the Creative Commons Attribution License, which permits unrestricted use, distribution, and reproduction in any medium, provided the original work is properly cited.

\begin{abstract}
Background. Sleep disorders are frequent nonmotor symptoms of Parkinson's disease (PD). Polysomnography (PSG) has been the gold standard for its assessment. However, it requires patients to stay overnight in a hospital or sleep center. The mobile twochannel electroencephalography (EEG)/electrooculography (EOG) recording system is a self-applicable and affordable method to objectively assess sleep at home. We aimed at evaluating patients with PD to confirm the difference in sleep parameters between the portable recording system and PSG. Methods. PSG and the portable recording system were simultaneously performed on a similar night in eight patients with PD. We compared the difference in sleep parameters between them using nonparametric tests. Results. All patients displayed a score of both PDSS $-2 \geq 15$ and PSQI $\geq 5$, respectively, which revealed poor sleep quality. There was no difference in the sleep parameters between the portable recording system and PSG, except for the percentage of sleep stage N3. Regarding the detection of REM sleep without atonia, we observed accordance between the portable recording system and PSG in six patients $(P=0.686)$. Conclusions. The portable EEG/EOG recording system may gain an advantage from home-based evaluations for habitual sleep at home. Our study on device validation may contribute to measuring natural sleep, including rapid eye movement (REM) sleep behavioral disorder (RBD), in an outpatient care setting.
\end{abstract}

\section{Introduction}

In Parkinson's disease (PD), sleep disorders are frequent nonmotor symptoms with an estimated occurrence rate of $74-88 \%[1,2]$, inducing sleep fragmentation and difficulties in maintaining sleep or falling asleep. Sleep fragmentation may increase the difficulties associated with PD pathology [3]. PD-related sleep disorders include nocturnal motor symptoms, psychosis, hallucinations, urinary incontinence, depression, cognitive impairment, vivid dreaming, and rapid eye movement (REM) sleep behavioral disorder (RBD). Since idiopathic RBD is the prodromal stage of PD [4] and $\mathrm{PD}$ with RBD has a more rapid progression with increased cognitive decline $[5,6]$, the importance of $\mathrm{RBD}$ has heightened.

Self-reported sleep diaries or questionnaires have been used extensively, with each method having its own strengths and limitations. To date, polysomnography (PSG) has been the gold standard assessment methodology to objectively measure the following conventional sleep parameters: sleep efficiency (SE), total sleep time (TST), wake time after sleep onset (WASO), and sleep onset latency (SOL). However, patients have to stay overnight in a hospital or sleep center for these tests. Thus, a selfapplicable and affordable method is needed to measure sleep at home. Researchers have investigated several biomedical, mechanical, or kinetic devices for home-based sleep monitoring and have obtained some accuracy [7]. For example, the Mobile Health Systems Lab Sleep Band system consists of eight-channel biosignal electrodes on the headband and demonstrates the typical non-REM sleep pattern [8]. Wireless single-channel headband sleep systems reportedly have a moderate to high agreement with PSG in healthy participants [9]. Nonetheless, they cannot detect REM sleep without atonia (RWA), which is required for diagnosing REM behavior disorder (RBD). The 
mobile two-channel electroencephalography (EEG)/electrooculography (EOG) recording system (SleepGraph ${ }^{\circledR}$, Proassist Co., Japan) is a self-applicable and affordable method to objectively assess sleep at home. The estimated sleep parameters were well correlated with those of PSG in a validation study of healthy adults $[10,11]$. Moreover, this system can detect RWA and objectively diagnose RBD. We evaluated patients with PD to confirm the difference in sleep parameters between the portable EEG/EOG recording system and PSG. Our study on device validation may contribute to measuring natural sleep, including $\mathrm{RBD}$, in an outpatient care setting.

\section{Methods}

We evaluated eight patients diagnosed with PD according to the International Parkinson and Movement Disorder Society (MDS) diagnostic criteria [12]. The clinical basic evaluations at the start of the study were the following: Hoehn-Yahr stage, MDS Revision of the Unified PD Rating Scale (UPDRS) parts 3 and 4 [13], subitem "psychosis" and "anxiety" on MDS-Non-Motor Rating Scale [14], Japanese version of the Montreal Cognitive Assessment (MoCA-J) [15], Japanese version of Parkinson's Disease Sleep Scale (PDSS)-2 [16], Japanese version of Sleep Behavior Disorder Screening Questionnaire (RBDSQ) [17], Pittsburgh Sleep Questionnaire Index (PSQI), and Beck depression score [18].

The portable recording system (SleepGraph ${ }^{\circledR}$, Proassist Co., Japan; medical device certification number: 231AHBZX00001000) consisted of a pair of bipolar EEG and EOG electrode leads, and the receiver was used for frontal EEG and EOG recording (Figure 1) [10, 11]. The forehead EEG was recorded from Fpl with the contralateral mastoid process (M2) as a reference. The EOG was recorded from two electrodes on the skin of opposing chin muscles approximately $1 \mathrm{~cm}$ below the eyes. The signal was recorded at a sampling frequency of $128 \mathrm{~Hz}$ using 0.540 and 0.544 EEG filters. Amplified and filtered analog data from the electrodes were converted into a digital signal using a 14-bit A/D converter, sent to a bedside-located receiver, and stored for offline data analysis. Sleep stage scoring was based on the forehead EEG signal [11], and in addition to the sleep stage structure, subsequent sleep measurements such as SE, TST, WASO, and SOL were calculated using the AASM rules [19]. When chin electromyography (EMG) activity, defined as the duration of phasic muscle activity lasting $0.1-5$ seconds with an amplitude four times greater than that of the background, is occupied by more than $50 \%$ for mini epochs for $3 \mathrm{~s}$, the epoch was defined as RWA. The ratio of RWA to total REM sleep (SREM) was calculated automatically.

2.1. Procedure. The PSG and the portable EEG/EOG recording system were simultaneously performed on the same night, and clinical evaluation was performed a few days prior. A standard PSG was performed and included six EEG signals (F3-M2, F4-M1, C3-M2, C4-M1, O1-M2, and O2M1), two channels of EOG signals (E1-M2 and E2-M2), chin electromyography (EMG) (EMG1-EMG2 and EMG1EMG3), and electrocardiography (ECG). The diagnostic

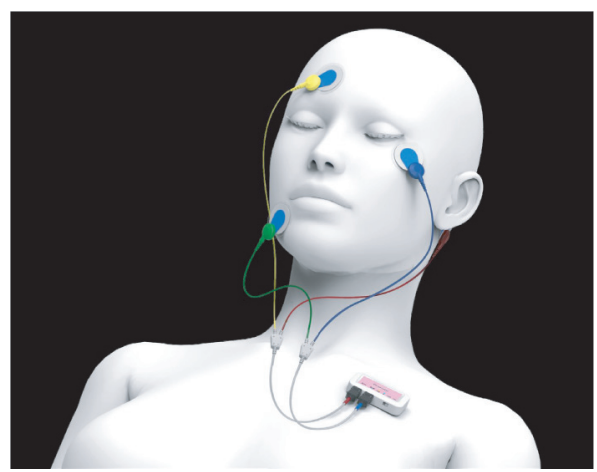

Figure 1: Portable recording system (ZA) (SleepGraph ${ }^{\circledR}$, Proassist Co., Japan).

PSG and portable EEG/EOG recording systems were manually scored by a certified sleep technologist with the definition of the AASM scoring manual [19]. The sleep technologist was not informed of any clinical information, except for age and gender. The study protocol was approved by the Medical Ethics Committee of the Nara Medical University.

2.2. Statistical Analysis. A comparison of the difference in sleep parameters between the portable EEG/EOG recording system and the PSG was performed using the nonparametric Mann-Whitney test and the Spearman correlation coefficient. The effect size was calculated using Cohen's $d$. Category was analyzed using the chi-square test. Statistical significance was set at $P<0.05$. SPSS software (version 24) was used for the statistical analysis.

\section{Results}

The basic clinical characteristics are shown in Table 1. Three and five patients were in the early and late stages, respectively, and all patients had motor complications. Five patients had mild cognitive impairment defined by the MoCA-J, and nontroublesome visual hallucinations were observed in five patients. One patient received continuous intrajejunal infusion of levodopa-carbidopa intestinal gel (LCIG) (levodopa/carbidopa: 44/10.1 mg/ $\mathrm{h}$ ), and seven patients were administered long-acting dopamine agonists. All patients had a score of both PDSS$2 \geq 15$ and PSQI $\geq 5$, which revealed poor sleep quality [20]. Six patients had mild depression, defined as 20 to 28 points on the BDI [21]. Five patients had a score of $\geq 5$ on the RBDSQ [17].

No difference was observed in sleep parameters between the portable EEG/EOG recording system and PSG, except for sleep stage N3 (Table 2 and Figure 2). Sleep efficiency, WASO, SOL, sleep period time (SPT), percentage of REM periods, and RWA/SREM on the portable EEG/EOG recording system were significantly correlated with PSG. As for the detection of RWA (Table 3), the accordance between the portable EEG/EOG recording system and PSG was found in six out of eight patients $(P=0.686)$. 
TABLE 1: Basic characteristics of subjects with Parkinson's disease.

\begin{tabular}{|c|c|c|c|c|c|c|c|c|}
\hline & Patient 1 & Patient 2 & Patient 3 & Patient 4 & Patient 5 & Patient 6 & Patient 7 & Patient 8 \\
\hline Age/gender & $67 / \mathrm{M}$ & $57 / \mathrm{M}$ & $66 / \mathrm{F}$ & $70 / \mathrm{F}$ & $61 / \mathrm{M}$ & $50 / \mathrm{M}$ & $78 / \mathrm{M}$ & $76 / \mathrm{F}$ \\
\hline Disease duration (years) & 5 & 11 & 11 & 12 & 11 & 10 & 10 & 17 \\
\hline Hypertension & - & - & - & + & - & - & - & - \\
\hline Body mass index & 20.5 & 19.4 & 20.2 & 22.6 & 27.2 & 19.4 & 21.7 & 16.1 \\
\hline Hoehn-Yahr stage & 2 & 2 & 2 & 4 & 4 & 4 & 4 & 5 \\
\hline MDS-UPDRS part 3 & 32 & 10 & 7 & 38 & 22 & 25 & 28 & 57 \\
\hline MDS-UPDRS part 4 & 5 & 11 & 4 & 7 & 7 & 7 & 4 & 7 \\
\hline MoCA-J & 25 & 23 & 26 & 22 & 25 & 29 & 26 & 23 \\
\hline Psychosis* & 0 & 2 & 2 & 11 & 4 & 0 & 3 & 0 \\
\hline Anxiety* & 2 & 12 & 0 & 3 & 17 & 6 & 6 & 2 \\
\hline Levodopa (mg/day) & 50 & 700 & 600 & 400 & 550 & LCIG & 1100 & 400 \\
\hline Dopamine agonists (DA) & + & + & + & + & + & - & + & + \\
\hline Long-acting DA & + & + & + & + & + & - & + & + \\
\hline Sleeping drug & - & + & - & + & + & - & - & + \\
\hline PDSS-2 total score & 17 & 32 & 21 & 21 & 20 & 27 & 43 & 36 \\
\hline Disturbed sleep on PDSS-2 & 10 & 15 & 14 & 13 & 9 & 14 & 15 & 10 \\
\hline Motor symptoms at night on PDSS-2 & 4 & 9 & 4 & 2 & 5 & 8 & 15 & 11 \\
\hline PD symptoms at night on PDSS-2 & 3 & 7 & 3 & 6 & 6 & 5 & 13 & 14 \\
\hline PSQI total score & 6 & 14 & 13 & 15 & 14 & 10 & 15 & 10 \\
\hline Beck depression score & 14 & 28 & 16 & 21 & 23 & 20 & 27 & 20 \\
\hline RBD score & 9 & 7 & 2 & 9 & 2 & 4 & 12 & 6 \\
\hline
\end{tabular}

MDS-UPDRS: Movement Disorder Society Revision of the Unified PD Rating Scale; LCIG: continuous intrajejunal infusion of levodopa-carbidopa intestinal; MoCA-J: Japanese version of the Montreal Cognitive Assessment; PDSS: PD Sleep Scale; PSQI: Pittsburgh Sleep Questionnaire Index; RBD: REM sleep behavior disorder. *MDS-Non-Motor Rating Scale.

TABLE 2: Difference of sleep parameters between polysomnography and the portable EEG/EOG recording system.

\begin{tabular}{|c|c|c|c|c|c|c|c|c|}
\hline & \multicolumn{2}{|c|}{ PSG } & \multicolumn{2}{|c|}{$\begin{array}{l}\text { Portable EEG/ } \\
\text { EOG recording } \\
\text { system }\end{array}$} & \multirow{2}{*}{$\begin{array}{c}\text { Mann-Whitney } \\
P\end{array}$} & \multirow{2}{*}{$\begin{array}{l}\text { Effect size } \\
\text { Cohen d }\end{array}$} & \multicolumn{2}{|c|}{ Spearman rank correlation } \\
\hline & Mean & $\mathrm{SD}$ & Mean & $\mathrm{SD}$ & & & $P$ & Correlation coefficient \\
\hline TST (min) & 381.8 & 58.9 & 394.1 & 103.9 & 0.753 & 0.15 & 0.693 & 0.167 \\
\hline TIB (min) & 644.1 & 56.8 & 673 & 65.6 & 0.371 & 0.47 & 0.568 & 0.24 \\
\hline SPT (min) & 507.2 & 95.8 & 508 & 92.8 & 1 & 0.01 & $0.01^{\dagger}$ & 0.833 \\
\hline SOL (min) & 89 & 81.3 & 94.7 & 77.2 & 0.793 & 0.07 & $0.001^{\dagger}$ & 0.929 \\
\hline WASO (times) & 30.7 & 9.7 & 25.5 & 12.3 & 0.246 & 0.47 & $0.003^{\dagger}$ & 0.896 \\
\hline WASO (min) & 117.8 & 50.1 & 136 & 69.4 & 0.6 & 0.3 & 0.058 & 0.69 \\
\hline WASO (\%) & 29.9 & 10.3 & 39.8 & 26.4 & 0.674 & 0.49 & $0.002^{\dagger}$ & 0.905 \\
\hline SE (\%) & 60.6 & 7.8 & 55.5 & 11.7 & 0.345 & 0.51 & $0.015^{\dagger}$ & 0.81 \\
\hline REM (\%) & 6.7 & 7.7 & 13.3 & 10.9 & 0.093 & 0.70 & $<0.001^{\dagger}$ & 0.976 \\
\hline N1 (\%) & 10.4 & 3.7 & 8.8 & 2.9 & 0.401 & 0.48 & 0.086 & 0.643 \\
\hline N2 (\%) & 52.7 & 6.4 & 55.9 & 10.5 & 0.401 & 0.37 & 0.12 & 0.595 \\
\hline N3 (\%) & 25 & 6.3 & 19.5 & 4.3 & $0.036^{\dagger}$ & 1.02 & 0.289 & 0.429 \\
\hline RWA (\%) & 5 & 6 & 1.9 & 1.8 & 0.293 & 0.70 & 0.435 & 0.323 \\
\hline RWA/total SREM (\%) & 37.3 & 36.1 & 17.1 & 13.6 & 0.431 & 0.74 & $0.028^{\dagger}$ & 0,762 \\
\hline
\end{tabular}

TST: total sleep time; TIB: time in bed; SPT: sleep period time; WASO: wake time after sleep onset; SOL: sleep onset latency; SE: sleep efficiency; EEG/EOG: electroencephalography/electrooculography, $\dagger: P<0.05$.

\section{Discussion}

The present study showed that TIB, SPT, WASO, SE, and REM period percentages were not significantly different between the portable EEG/EOG recording system and PSG. Also, a significant correlation was found between these sleep parameters, similar to previous validation studies of healthy subjects $[10,11]$. The TST and sleep stage $\mathrm{N} 1$ and $\mathrm{N} 2$ results did not show a significant correlation with the Spearman correlation coefficient, and there was a significant difference for stage N3 using the Mann-Whitney test. From the validation study of the portable EEG/EOG recording system, a correlation for stages $\mathrm{N} 1, \mathrm{~N} 2$, and $\mathrm{N} 3$ between two independent scorers was not consistent on Bland-Altman plots, and a dissociation of the $P$ value between the two scorers was observed for these stages [11]. K complex and delta waves that characterize stages $\mathrm{N} 2$ and $\mathrm{N} 3$ can be detected from the electrodes located in the frontal lobe region $[22,23]$, but alpha and sleep spindle waves are more clearly observable in the central and occipital EEG [22-25]. Thus, the forehead EEG electrodes have less power to detect certain characteristic EEG waves which identify sleep stages. 


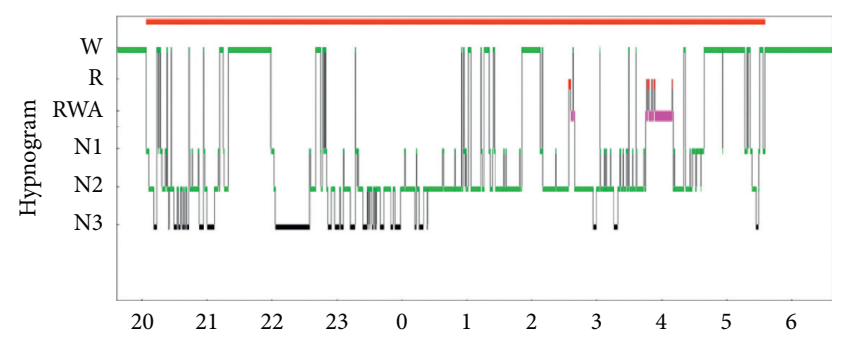

(a)

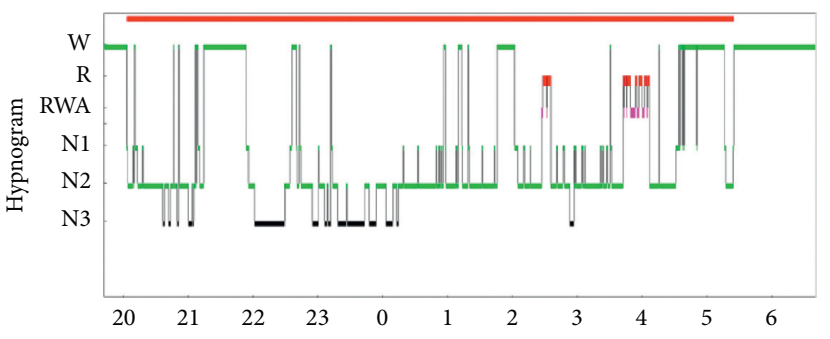

(b)

Figure 2: Hypnogram. (a) Polysomnography. (b) Portable recording system (ZA) (SleepGraph ${ }^{\circledR}$, Proassist Co., Japan).

TABLE 3: Detection of REM sleep without atonia (RWA).

\begin{tabular}{|c|c|c|c|c|c|c|c|c|}
\hline & Patient 1 & Patient 2 & Patient 3 & Patient 4 & Patient 5 & Patient 6 & Patient 7 & Patient 8 \\
\hline Polysomnography & + & + & + & + & - & + & + & + \\
\hline Portable EEG/EOG recording system & + & + & + & + & + & - & + & + \\
\hline RBD score $\geq 5$ & + & + & - & + & - & - & + & + \\
\hline
\end{tabular}

RBD: rapid eye movement (REM) sleep behavioral disorders.

The importance of RBD has been increasing from the aspect of the prodromal stage of neurodegenerative diseases such as PD and the prediction of disease progression [5]. For example, PD patients with RBD have a higher incidence of dementia or worse autonomic dysfunction $[6,26]$. A strength of the present study is that the portable EEG/EOG recording system can detect RWA, although the percentage of RWA seems to be slightly different between the portable EEG/EOG recording system and PSG. The diagnosis of RBD is recognized worldwide and requires the detection of RWA on PSG. However, PSG is labor-intensive, time-consuming, and expensive [27], and the subject had to stay overnight in a laboratory setting. Due to the highly consistent detection of RWA on the portable EEG/EOG recording system and PSG, it is expected that RWA in addition to natural sleep can be evaluated in outpatients. A discordance of RWA detection between the portable EEG/EOG recording system and PSG was observed in two patients, but they did not have a score $\geq 5$ on the RBDSQ. The limitations of the present study are the small sample size and the recording of results being undertaken by a single expert sleep scorer. Fewer electrodes in the portable recording system than that in PSG may have led to an error in diagnosis, such as phasic-activity type RWA.

In conclusion, sleep parameters between the portable EEG/EOG recording system and PSG are likely to be similar rather than sleep stages $\mathrm{N} 1, \mathrm{~N} 2$, and N3, and this may gain an advantage from home-based evaluations for habitual sleep at home, particularly in subjects who are difficult to evaluate otherwise. The two-channel portable EEG/EOG recording system may be a suitable technique for diagnosing $\mathrm{RBD}$, especially in an outpatient care setting.

\section{Data Availability}

The data used to support the findings of this study are available from the corresponding author upon request.

\section{Ethical Approval}

The study protocol was approved by the Medical Ethics Committee of Nara Medical University (no. 2710).

\section{Consent}

The authors obtained written informed consent from all patients.

\section{Conflicts of Interest}

The authors report no conflicts of interest including Proassist Co., Japan, related with the present manuscript.

\section{Authors' Contributions}

$\mathrm{H}$ Kataoka and $\mathrm{T}$ Takatani were responsible for the overall study design and contributed to organization, planning, and coordination of the study, to running the study and acquisition of data, and to analysis and interpretation of data. $\mathrm{H}$ Kataoka wrote the manuscript. H Kataoka and K Sugie contributed to the drafting and critical revision of part of the submitted materials.

\section{Acknowledgments}

The authors are extremely grateful to the corporation of all people who participated in the present study. In particular, Sayomi Yamamoto, Division of Central Clinical Laboratory, Nara Medical University, performed the setting of polysomnography and the portable recording system (ZA) (SleepGraph ${ }^{\circledR}$, Proassist Co., Japan), and Proassist Co., Japan, will rent 2 sets of the portable recording system (SleepGraph ${ }^{\circledR}$, medical device certification number: 231AHBZX00001000). This study was funded by Proassist Co., Japan, via Nara Medical University. 


\section{References}

[1] S. A. Factor, T. McAlarney, J. R. Sanchez-Ramos, and W. J. Weiner, "Sleep disorders and sleep effect in Parkinson's disease," Movement Disorders, vol. 5, no. 4, pp. 280-285, 1990.

[2] W. G. H. Oerlemans and A. W. de Weerd, "The prevalence of sleep disorders in patients with Parkinson's disease," Sleep Medicine, vol. 3, no. 2, pp. 147-149, 2002.

[3] S. Sohail, L. Yu, J. A. Schneider, D. A. Bennett, A. S. Buchman, and A. S. P. Lim, "Sleep fragmentation and Parkinson's disease pathology in older adults without Parkinson's disease," Movement Disorders, vol. 32, no. 2, pp. 1729-1737, 1990.

[4] A. Al-Qassabi, "Sleep disturbances in the prodromal stage of Parkinson disease," Current Treatment Options in Neurology, vol. 19, no. 6, 22 pages, 2017.

[5] S.-M. Fereshtehnejad, S. R. Romenets, J. B. M. Anang, V. Latreille, J.-F. Gagnon, and R. B. Postuma, "New clinical subtypes of Parkinson disease and their longitudinal progression," JAMA Neurology, vol. 72, no. 8, pp. 863-873, 2015.

[6] R. B. Postuma, J.-A. Bertrand, J. Montplaisir et al., "Rapid eye movement sleep behavior disorder and risk of dementia in Parkinson's disease: a prospective study," Movement Disorders, vol. 27, no. 6, pp. 720-726, 2012.

[7] P. Leelaarporn, P. Wachiraphan, T. Kaewlee et al., "Sensordriven achieving of smart living: a review," IEEE Sensors Journal, vol. 21, no. 9, pp. 10369-10391, 2021.

[8] M. L. Ferster, C. Lustenberger, and W. Karlen, "Configurable mobile system for autonomous high-quality sleep monitoring and closed-loop acoustic stimulation," IEEE Sensors Letters, vol. 3, no. 5, pp. 1-4, 2019.

[9] J. R. Shambroom, S. E. Fábregas, and J. Johnstone, "Validation of an automated wireless system to monitor sleep in healthy adults," Journal of Sleep Research, vol. 21, no. 2, pp. 221-230, 2012.

[10] R. Kanemura, H. Sumitani, M. Matsuo et al., "Evaluation of a portable two-channel electroencephalogram monitoring system to analyze sleep stages," Journal of Oral and Sleep Medicine, vol. 2, no. 2, pp. 101-108, 2020.

[11] S. Nonoue, M. Mashita, S. Haraki et al., "Inter-scorer reliability of sleep assessment using EEG and EOG recording system in comparison to polysomnography," Sleep and Biological Rhythms, vol. 15, no. 1, pp. 39-48, 2007.

[12] R. B. Postuma, D. Berg, M. Stern et al., "MDS clinical diagnostic criteria for Parkinson's disease," Movement Disorders, vol. 30, no. 12, pp. 1591-1601, 2015.

[13] C. G. Goetz, B. C. Tilley, S. R. Shaftman et al., "Movement disorder society-sponsored revision of the unified Parkinson's disease rating scale (MDS-UPDRS): scale presentation and clinimetric testing results," Movement Disorders, vol. 23, no. 15, pp. 2129-2170, 2008.

[14] K. R. Chaudhuri, A. Schrag, D. Weintraub et al., "The movement disorder society nonmotor rating scale: initial validation study," Movement Disorders, vol. 35, no. 1, pp. 116-133, 2020.

[15] Y. Fujiwara, H. Suzuki, M. Yasunaga et al., "Brief screening tool for mild cognitive impairment in older Japanese: validation of the Japanese version of the Montreal Cognitive Assessment," Geriatrics and Gerontology International, vol. 10, no. 3, pp. 225-232, 2010.

[16] K. Suzuki, M. Miyamoto, T. Miyamoto et al., "Nocturnal disturbances and restlessness in Parkinson's disease: using the Japanese version of the Parkinson's disease sleep scale-2,"
Journal of the Neurological Sciences, vol. 318, no. 1-2, pp. 76-81, 2012.

[17] T. Miyamoto, M. Miyamoto, M. Iwanami et al., “The REM sleep behavior disorder screening questionnaire: validation study of a Japanese version," Sleep Medicine, vol. 10, no. 10, pp. 1151-1154, 2009.

[18] A. T. Beck, R. A. Steer, and G. K. Brown, Manual for the Beck Depression Inventory, The Psychological Association, San Antonio, TX, USA, Second edition, 1996.

[19] R. B. Berry, C. L. Albertario, and S. M. Harding, For the American Academy of Sleep Medicine. The AASM Manual for the scoring of sleep and associated events: rules, terminology and technical specifications, Version 2.5, American Academy of Sleep Medicine, Darien, IL, USA, 2018.

[20] K. Suzuki, T. Miyamoto, M. Miyamoto et al., "Evaluation of cutoff scores for the Parkinson's disease sleep scale-2," Acta Neurologica Scandinavica, vol. 131, no. 6, pp. 426-430, 2015.

[21] A. T. Beck, R. A. Steer, and M. G. Carbin, "Psychometric properties of the Beck depression inventory: twenty-five years of evaluation," Clinical Psychology Review, vol. 8, no. 1, pp. 77-100, 1988.

[22] L. McCormick, T. Nielsen, A. Nicolas, M. Ptito, and J. Montplaisir, "Topographical distribution of spindles and K-complexes in normal subjects," Sleep, vol. 20, no. 11, pp. 939-941, 1997.

[23] N. Dehghani, S. S. Cash, and E. Halgren, "Topographical frequency dynamics within EEG and MEG sleep spindles," Clinical Neurophysiology, vol. 122, no. 2, pp. 229-235, 2011.

[24] A. Compston, "The Berger rhythm potential changes from the occipital lobes in man," Brain, vol. 133, no. 1, pp. 3-6, 2010.

[25] C. Marzano, F. Moroni, M. Gorgoni, L. Nobili, M. Ferrara, and L. De Gennaro, "How we fall asleep: regional and temporal differences in electroencephalographic synchronization at sleep onset," Sleep Medicine, vol. 14, no. 11, pp. 1112-1122, 2013.

[26] R. B. Postuma, J.-F. Gagnon, M. Vendette, K. Charland, and J. Montplaisir, "Manifestations of Parkinson disease differ in association with REM sleep behavior disorder," Movement Disorders, vol. 23, no. 12, pp. 1665-1672, 2008.

[27] D. J. Levendowski, D. Popovic, C. Berka, and P. R. Westbrook, "Retrospective cross-validation of automated sleep staging using electroocular recording in patients with and without sleep disordered breathing," International Archives of Medicine, vol. 5, no. 1, p. 21, 2012. 